\title{
A Shoulder Pad Insert Vibrotactile Display
}

\author{
Aaron Toney ${ }^{\dagger}$, Lucy Dunne ${ }^{\ddagger}$, Bruce H. Thomas ${ }^{\dagger}$, Susan P. Ashdown \\ ${ }^{\dagger}$ Wearable Computer Laboratory \\ School of Computer and Information Science \\ University of South Australia \\ aaron.toney@hhhh.org,
}

bruce.thomas@unisa.edu.au

Abstract

Touch is the most intimate and inherently private human sense and provides the potential for discrete, low social weight human computer interaction. This paper presents initial research findings on issues of integrating a vibrotatcitle display and support electronics into a standard clothing insert, the shoulder pad. Research on construction methods is presented along with a discussion of the merits and drawbacks of each technique. User study data for response to tactile display stimuli, collected with a varying number of stimulators, is then presented with initial conclusions as to the type and format of data suitable for shoulder based tactile arrays.

\section{Introduction}

An examination of basic clothing structure reveals a new area of investigation for wearable computer designers; that of standard clothing inserts. We define clothing inserts as layers of padding, interfacing or other materials meant to give shape, strength or protective function to the garment. This definition is broad enough to include garment pads such as shoulder, knee or elbow pads, as well as less physically obvious inserts such as the layers of interfacing added to stiffen garment sections (e.g. the waistband, shoulder area, collar, or lapel). The added stiffness and bulk of these inserts provides a ready-made space in which small-scale electronics can be housed without visually changing the garment. Integration of electronics in these areas requires investigation of the wear stresses that will be placed on the electronics, as well as any discomfort caused by hard components at close proximity to the body.

\subsection{The Problem}

Clothing is an elemental and pervasive part of human life. Successful integration of electrical technologies into clothing must address the culture, tradition, and technology inherent to clothing forms. Wearable electronic design requires consideration not only of function, but of comfort, mobility and social weight. Social Weight[19] is a measure of the degradation of social interaction that occurs between the user and other people caused by the use of an item of technology. For example, a ringing cell phone imposes a very high social weight when a phone conversation interrupts another social interaction, whereas a digital watch that vibrates softly to mark the hour imposes almost no social weight at all. The design of wearable devices must operate within the confines

of the current acceptable concepts of "clothing". This paper seeks to address how best to incorporate tactile displays into clothing while remaining socially acceptable and providing useful bandwidth to the user.

\subsection{Objectives}

Integration of electronics into garment inserts presents new opportunities for location-appropriate electronics. The proximity of the clothing layer to the body provides a convenient location for tactile displays, and the sense of touch holds promise to be an effective channel of communication in wearable computing. This work seeks to initially characterize shoulder worn vibrotactile displays. Data required for the selection of optimal type, number and location of stimulators as well as perceptible bandwidth of information to the user are sought. In our studies of integration of a wearable computer into a traditional business suit [19], tactile input meets several important goals: it is discreet, delivering information to the user through a low social weight channel, and it presents information to the user with lower cognitive loads as compared with other sensory channels. As a result tactile displays hold out the promise for low social weight[19] devices.

The shoulder pad in particular was chosen as a highly useful garment insert because of its common integration into the standard business suit, one of the most culturally pervasive garments in western society. The business suit is also commonly worn by individuals who are likely to have an interest in mobile access to information. The shoulder pad can exist in many physical forms, most notably variations in shape and size. Most suits (both men's and women's) contain some degree of stabilization in the shoulder area; however the height and weight of the stabilization may vary with style and current fashion trends.

The choice of integrating a vibrotactile display into a standard shoulder pad insert meets the previously mentioned and several other goals: 1) the shoulder pad insert makes use of an existing volume within standard garments, allowing the designer to integrate electronics without changing the outward appearance of the garment; 2) the position allows the display to mimic social conventions such as tapping on the shoulder area for alerts or guidance; 3 ) the components that will not survive washing or dry cleaning are integrated into a garment insert that is conventionally removable during cleaning procedures; and 4) the shoulder pad insert vibrotactile display maintains the function and feel of the 
garment with the integrated electronics without impacting the user's mobility or comfort. To our knowledge this work represents the first effort to place an array of simulators on the shoulder area.

\section{The Sense of Touch}

This section will review tactile sensory perception and the mechanoreceptors that facilitate its various modalities.

\subsection{Mechanoreceptors}

Within the skin, many different mechanoreceptors facilitate the sense of touch. Table 1 outlines seven mechanoreceptors and their sense modality. An analysis of each modality reveals different levels of appropriateness for stimulation using a clothing insert based tactile display.

\begin{tabular}{|l|l|}
\hline Receptor & Sense modality \\
\hline Meissner Corpuscle & Stroking, fluttering \\
\hline Merkel Disk Receptor & Pressure, texture \\
\hline Pacinian Corpuscle & Vibration \\
\hline Ruffini Ending & Skin stretch \\
\hline Hair follicle & Stroking, fluttering \\
\hline Hair & Light stroking \\
\hline
\end{tabular}

Table 1: Human mechanoreceptors and corresponding sensory modalities

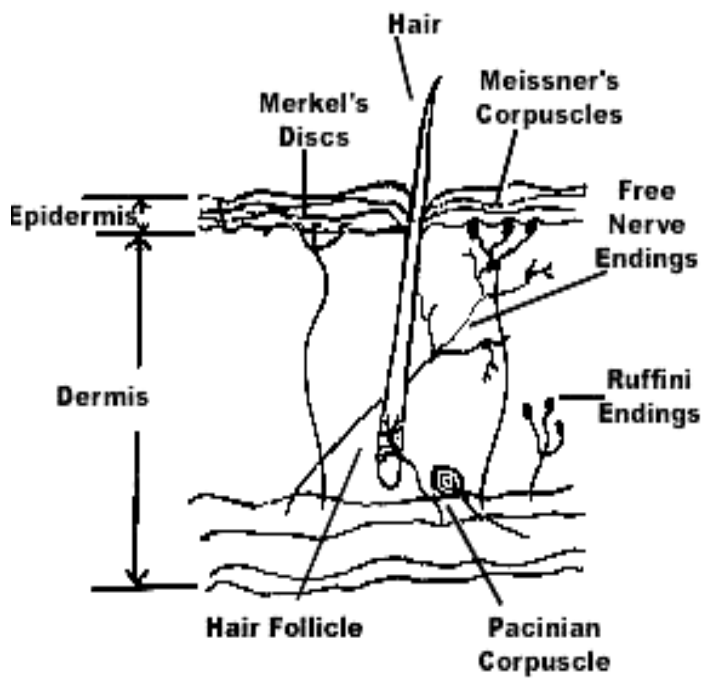

Figure 1: Sensory Anatomy of the skin

Vibration: Vibration is a likely candidate for clothing insert based tactile displays as the scale of the impulse and geometry of a vibration device facilitate easy integration into small garment spaces. The Pacinian corpuscles are the mechanoreceptors responsible for most detection of vibrational stimuli. Pacinian corpuscles are some of the deepest mechanoreceptors in the dermis, and are the largest touch receptors. They are the fastest-adapting of the class of fast-acting receptors, meaning they respond quickly to changing stimuli. They have optimal sensitivity around 400 $\mathrm{Hz}$, and have difficulty detecting frequencies below $50 \mathrm{~Hz}$ or above $600 \mathrm{~Hz}$ [26].
Pressure and Stroking: Although vibration is merely the time variance of pressure about an equilibrium level, it remains a distinct dermal sensory channel from both pressure and stroking. Pressure and stroking communication channels both require the accurate generation of static or low time variant pressure distribution across the skin surface. In our application, the garment weight alone supplies a counter force for the stimulators, a force which is not constant enough to support reliable pressure or stroking stimulus.

Skin Stretch: While displays using the perception of stretch have been demonstrated[9], they inherently require at least two points of firm contact with the skin to operate, which are drawn apart to stretch the skin that they are in contact with. Any fabric between the skin and the stimulator may cause the contact points to slip. As a result stretch makes a poor mobile or garment-integrated communications channel.

Texture, Stroking, and Fluttering: The sensations of texture, light stroking, and fluttering are subtle sensations that are only perceptible directly on the skin surface. As these tactile channels are all severely muted when felt through clothing they are not an appropriate foundation for a wearable tactile displays.

\subsection{Sensory perception}

Prior research indicates that the smallest of situational changes effect sensory perception, including tactor area [15], amplitude or frequency[23], and body location[22, 24]. There has been little prior research in tactile perception in the shoulder area, so information on the effects of different variables on perception of vibration in the shoulder area is not available. For instance, the textile layers between the skin and the tactor in our application present variables not addressed in previous research.

\section{Selecting a Vibrotactile Stimulator}

The current vibrotactile technology solutions that might be appropriate for wearable applications are solenoids, speakers, piezoelectric actuators and electromagnetic motors. After a review of the properties of each solution, the authors chose to use a pancake motor based stimulator.

\subsection{Solenoids}

Solenoids small enough for use in wearable applications have found application in the construction of Braille displays[1] as well as main stream commercial human computer interface (HCI) applications[12]. The maximum firing frequency of solenoids is limited by the mechanical travel of the solenoid "slug". As a result solenoids would not be able to display across the desired range of 50-600 Hz. To function properly these small solenoids rely on a small sharp contact surface (one with a high degree of contrast) striking the skin, and garment layers between the solenoid and the skin heavily mute contact.

\subsection{Speakers and Piezoelectric Actuators}

A variety of speakers have been used to provide 
vibrotatile display for wearable applications. The speakers used range from rather large conventional speakers with a diameter of $101.6 \mathrm{~mm}$ [20], to much smaller electromechanical and piezoelectric devices with diameters of $25.4 \mathrm{~mm}[5,8,16]$ or less. In addition to having a small diameter both piezoelectric and electromechanical elements have the benefit of being very thin at approximately $1 \mathrm{~mm}$ and $3 \mathrm{~mm}$ respectively. Both actuators have a stimulation frequency range over the range appropriate for tactile perception.

The development of motors for use in pagers and cellular phones has resulted in significant decreases in the size, power consumption and price of electromagnetic motors. While motors were ultimately chosen for our investigations the authors feel that, aided by the refinement of commercialization, piezoelectric stimulators will ultimately prove more useful for clothing integrated tactile displays. Piezoelectric stimulators have previously been demonstrated in wearable applications $[4,5,8]$ and they are commercially available, thin, small, and flexible. Currently however all the piezoelectric stimulators known to the authors require more elaborate mounting topologies and higher operational voltages then their equivalent electromagnetic counterparts. The higher operational voltages required by piezoelectric materials create additional safety concern over mechanically equivalent low voltage motor based stimulators.

\subsection{Electromagnetic Motors}

The ability to deliver significant vibrational force at low voltages in a robust package has made motor based tactile stimulators a very appealing option to wearable researchers. As a result they have already been used in numerous wearable designs to provide vibrotactile stimulus[14, 16-19].

In initial subjective evaluations, current commercially available electromagnetic motors were able to provide substantially more vibrational force then comparably priced and sized piezoelectric stimulators. As a shoulder based display will have the hang weight of the garment bearing down upon it, the actuators must have the power to function under this load.

Motors also benefit from being easy to drive as they are activated by the simple application of voltage. The voltage signal applied can be digital, merely to spin the motor up, or analog for more subtle control. Motors generate a relatively high level of vibration when compared to other vibration generating technologies. Small counterweighted electromagnetic motors are packaged in two different configurations: cylindrical and pancake motors.

The cylindrical motors are miniature DC brush motors with a cam shaped counterweight. These motors typically range from 4-6mm in diameter, $15-20 \mathrm{~mm}$ in length, and draw $60-120 \mathrm{~mA}$ at $1.5-3 \mathrm{~V}$ depending on vendor and type. Our initial proof of concept prototype was designed around the cylindrical pager motors that were quickly abandoned in favor of pancake motors that deliver a more appropriate signal for our use.

The pancake motors trade height for increased diameter, and provide a more radially uniform distribution of vibrational energy whereas the cylindrical motors distribute most of their mechanical energy along the central axis of their body cylinder. After using both package types it seems to the authors that the pancake motors make less noise. Our current designs use the Sanko Electric 1E120 pancake motor.

\section{Output Tactile Display Devices}

Previous work of researchers in tactile perception has characterized parameters of most of the body with respect to its typical size, shape, and tactile threshold sensitivities[21, 26]. While some work has been done investigating optimal shape of shoulder based wearable modules[6], the shoulder has been largely neglected by prior work. To date few applications have used the shoulder as a target area for a tactile communications channel.

\subsection{Vibrotactile Arrays}

Vibrotactile displays on other parts of the body have already demonstrated a wide range of cognitive aids to improve situational awareness, navigation[18], reckoning, balance[25], and to decrease confusion about spatial and directional orientation [16].

The few applications that have incorporated the shoulder as a tactile display space have almost exclusively investigated applications for assistive technology for the disabled [10,25]. These applications all used a single actuator typically placed on the upper arm.

Osamu Morikawa's HyperMirror[14] used a shoulder worn single stimulator display to provide videoconference participants a vibrotactile cue for getting another person's attention. This cue literally provided a remote participant the ability to tap another videoconference participant at a remote location on the shoulder. The work is particularly relevant as it is the only work known to the authors, other than their own[19], to have used shoulder mounted pager motors. Morikawa's "Shoulder Tapping" interface, pictured in Figure 2(a), is composed of two curved sheets of plastic joined to form a shoulder mount. Set within the shoulder mount is a cylindrical pager motor. Morikawa used two such devices were used to provide the user with a stereo tactile display.

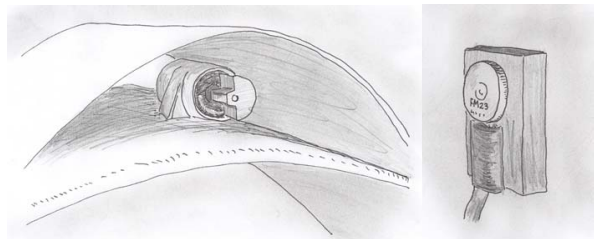

Figure 2: (a) Morikawa's HyperMirror Actuator (b) Lindeman's Tactor.

Wearable vibrotactile arrays are uncommon, but have been demonstrated on the forearm[24], back[18, 20], and torso[15]. The two most commonly implemented vibrotactile arrays are located in the back of a vest $[17,18]$ or chair[11]. 
Both of these arrays are similar in construction and are implemented using either a $3 \times 3$ or $4 \times 4$ grid of pager motors. The implementation of one such chair based array proves instructional for the design of shoulder pads. The configuration used by Lindeman et al for their Near-Field Haptic display used the same style motors that were chosen for our current work. Their stimulator, or "Tactor", pictured in Figure 2(b) used small foam blocks to increase the overall stimulator area.

The application of vibrotactile displays is in its infancy. This study is undertaken to improve future garment based displays for wearable computing. Such improvements would not only enable advances in the authors previous work [3, 19] but create a diverse array of new applications.

\section{Development of Shoulder Pad}

\subsection{Objectives}

The objective for this project was to develop a tactile display contained within a standard shoulder pad that could present a stimulus to the user. More specifically, the display needed to be capable of presenting several distinct stimuli in multiple locations at once, and it needed to maintain the functions of a shoulder pad: shape, stability, and flexibility. An additional objective was to consider the range of variation of body size in the population in order to provide pads with appropriate contact for every shoulder configuration. The development challenges are to determine an appropriate range of sizes and to identify materials and construction methods that will fill these objectives

\subsection{Sizing/development of physical space}

To determine the appropriate shape of the shoulder pad and the number of sizes that would be necessary, analysis of the available space in the shoulder and the anthropometric variation of this area across the population was required. In the application of vibrotactile units it is especially important that the electronic piece fit the body closely. Given the variation of the population it was necessary to introduce size variation to optimize fit and function. In order to determine the available space on the shoulder and the number of sizes that would be necessary to fit the entire population, we first analyzed several measurements from the ANSUR[7] database of anthropometric measures (a survey of the body measurements of 3,982 subjects, 1,774 male and 2,208 female US Army personnel). Because of size restrictions and fitness requirements of the military, this database does not represent the anthropometric variation in the population as a whole, but does represent a large portion of the population.

The measures we were interested in deriving from the database were shoulder length, vertical distance from the horizontal plane, and shoulder curve length. These measurements are illustrated in Figure 3.

To obtain these measurements, we used the ANSUR measurements for shoulder length, cervicale height, acromial height and axilla height. These measurements are illustrated in Figure 4.

The shoulder length measurement was the only measure that was available directly from the database; all other measurements were derived from related measurements. The maximum vertical distance for a shoulder pad for each subject was derived by subtracting the acromial height measurement from the cervicale height measurement. This measure gives us the vertical height that would produce an absolutely horizontal line from the base of the neck out to the end of the shoulder. To obtain the pad curve length, we subtracted the axilla height from the acromial height. The result was multiplied by two, for the front and back curve. The measurements used to derive this are linear measures taken with an anthropometer, not curved measures following the curve of the body, but the added length generated by the slight curvature was not significant. The pad curve measure determines the underside length of the pad at the edge of the shoulder.

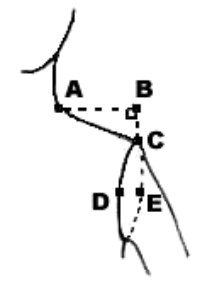

Figure 3: Desired dimensions for shoulder pad

Shoulder length A-C, vertical distance B-C, and curve length D-C-E

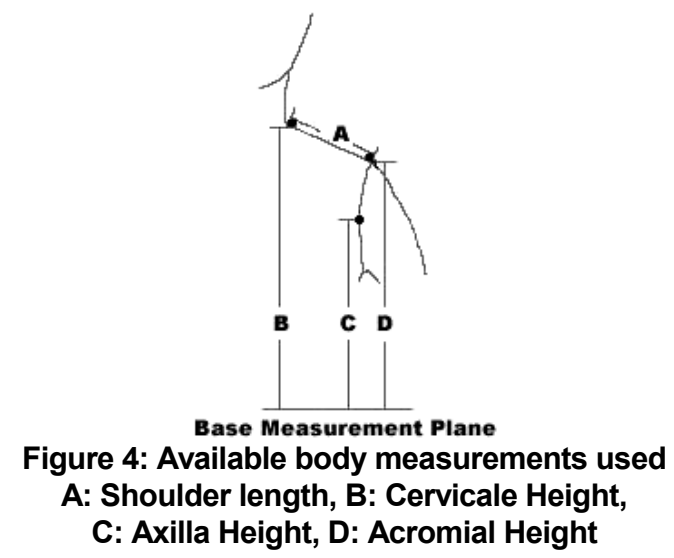

The derived measurements (pad height, pad curve length) were then analyzed to determine the number of sizes needed to fit the entire population. Two statistical representations were generated, histograms of each measure and correlations of length versus height. Length was used in the correlation because it is the most important sizing measure in a shoulder pad. As the pad tapers to nothing at the front hollow and back shoulder blade of the body, a too long or too short shoulder curve dimension has few adverse effects. An incorrect shoulder length measure has a more significant visual effect. A pad that is too short results in a collapsed line in the silhouette of the shoulder. A pad that is too long results in a 
protruding edge at the sleeve cap.

The correlation of length versus height resulted in a fairly strong relationship between the variables. The correlation coefficient is 0.667 , significant at the 0.01 level. The scatterplot is shown in Figure 5.

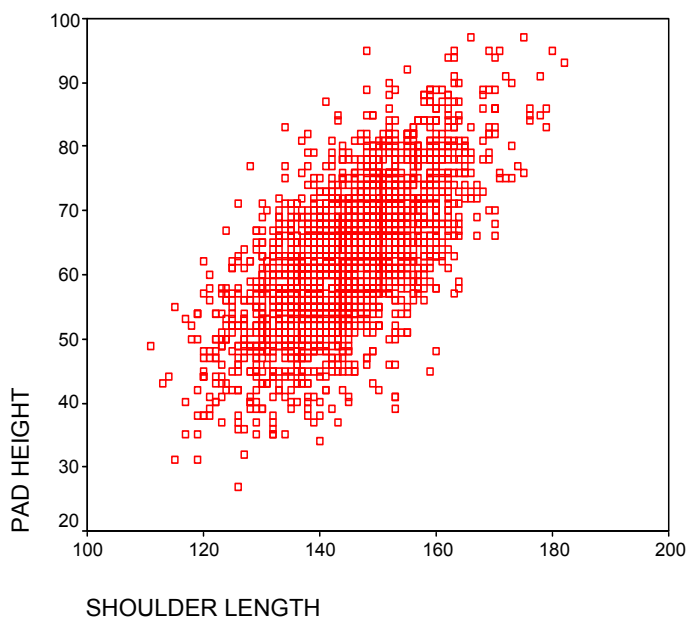

Figure 5: Scatter-plot of pad length versus pad height

Using the histograms of each measure, it was determined that the entire population could be effectively divided into 5 sizes. Eliminating the outlying extra small and extra large sizes left 3 sizes that could be expected to fit approximately $93 \%$ of the population. For the initial study we constructed pads appropriate for testing those three main groups representative of the small, medium, and large segments of the population. These sizes are shown in Table 2. Small outliers represented approximately $6 \%$ of the population while large outliers represented less than $1 \%$. The body sizes for each pad used to fit the population are shown in Table 2.

\begin{tabular}{|l|l|l|l|}
\hline SIZE & LENGTH & HEIGHT & CURVE \\
\hline 1 & $13 \mathrm{~cm}$ & $5 \mathrm{~cm}$ & $22 \mathrm{~cm}$ \\
\hline 2 & $15 \mathrm{~cm}$ & $7 \mathrm{~cm}$ & $24 \mathrm{~cm}$ \\
\hline 3 & $17 \mathrm{~cm}$ & $9 \mathrm{~cm}$ & $26 \mathrm{~cm}$ \\
\hline
\end{tabular}

Table 2: Body sizes for shoulder pad grade

The actual shoulder pad dimensions were then determined by subtracting standard ease values of $2 \mathrm{~cm}$ from the shoulder length and pad curve length (to allow for wearing ease) and 2 $\mathrm{cm}$ from the height (to allow the shoulder line to slope down from the horizontal). A test of these sizes led to further reductions in height to create a more visually acceptable silhouette. The largest pad was reduced in height by an additional centimeter to further reduce the bulk of this pad. The resulting grade for the shoulder pads is shown in Table 3 .

\begin{tabular}{|l|l|l|l|}
\hline SIZE & LENGTH & HEIGHT & CURVE \\
\hline 1 & $11 \mathrm{~cm}$ & $3 \mathrm{~cm}$ & $20 \mathrm{~cm}$ \\
\hline 2 & $13 \mathrm{~cm}$ & $4 \mathrm{~cm}$ & $22 \mathrm{~cm}$ \\
\hline 3 & $15 \mathrm{~cm}$ & $5 \mathrm{~cm}$ & $24 \mathrm{~cm}$ \\
\hline
\end{tabular}

Table 3: Shoulder pad grade

\subsection{Analysis of component materials/development}

The concept for embedding electronics into this type of garment insert centers on the use of the padding already present in the insert to provide both structure and support that protects the electronics and conceals their shape. For this application, we first experimented with a layering technique of latex rubber and muslin. This material solution was discarded because of its long drying time.

We next investigated the use of flexible urethane foam. The foam used was Flex-foam from Smooth-on, which was chosen for its ability to be easily molded into a shape and for its similar physical properties to materials used in conventional shoulder pads. Unfortunately activating a motor within this foam shape caused the foam to mechanically couple with the vibrating motor, spreading the vibrational stimulus to other areas of the pad. In our particular application it was necessary that the subjects be able to distinguish between motors and localize where vibration was initiated. Transmission of vibration through the pad when a motor was active made it difficult to distinguish motors. The use of foam does merit further investigation, using a vibration isolating foam in combination with the flex-foam.

A second prototype was made by mounting the motors on a thin $(0.318 \mathrm{~mm})$ polyester jersey knit base and creating the shoulder pad shape using polyester fiberfill, an amorphous mass of polyester fibers used in many apparel and home furnishing applications. The fiberfill performed very well at absorbing vibration and therefore isolating the sensation of each individual motor. However, the fiberfill was too soft to add structure to a garment, the primary function of a shoulder pad: it created the necessary volume for a shoulder pad, but not the structure or shape.

To compromise between these properties, the next prototype was constructed out of multiple layers of polyester and cotton batting. The polyester batting has a thickness of $1.74 \mathrm{~mm}$, and a loose construction of fibers which are needlepunched to tangle them and then covered with a light scrim of melt-blown fibers (fibers which are blown together as they are formed, melting them into a loose mesh). This structure isolates the vibration but also provides structure for the pad. Two layers of this batting were placed directly on top of the motors, which were affixed to the jersey knit fabric shell of the pad. Two layers of cotton batting, denser padding of similar thickness $(1.75 \mathrm{~mm})$, composed of cotton fibers needle-punched through a thin inner layer, were pad-stitched together and placed on top of the pad to create the curved shoulder shape and provide the additional structure. Pad stitching is a tailoring technique used to join layers of fabric together to create a shape but also maintain a flexible structure. The wires supplying power to each motor were coiled slightly within the pad, to help eliminate the transmission of vibration through the wires. This prototype proved most functional. It allowed the motors to vibrate independently, while providing the desired volume, shape, and structure for a shoulder pad. 


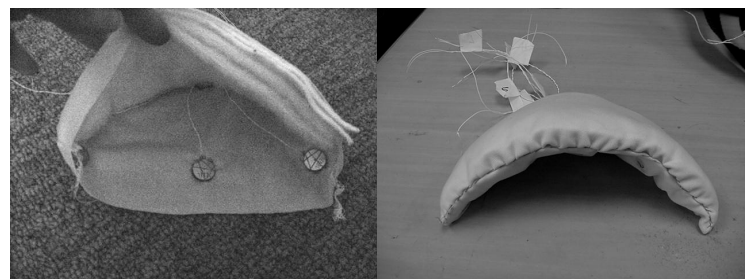

Figure 6: Final Prototype Layered Batting Construction a) Interior of pad showing layers, 4-motor configuration b) pad closure, showing curvature.

\section{Development of electronics}

Upon review of the literature an initial estimate of the shoulder's two point threshold, or the minimum perceivable distance between two stimulation points, was made at $38 \mathrm{~mm}$. This estimate was made based on two-point threshold data for the torso. Given the overall display space presented by our smallest shoulder pad, and using packing topologies where motor distribution is both uniform and just inside our estimate of the two point threshold, seven motors per shoulder pad was considered to be the greatest feasible number of motors. Flexibility as to the number and location of motors per shoulder pad was required. To achieve this all shoulder pads were constructed with a standard DIN9 connector interface using a consistent motor to pin number mapping that could support up to 8 motors and serve either as a right or left shoulder pad.

To facilitate initial testing a simple battery powered "button-box" (Figure 7a) was constructed to drive the motors. The authors felt it desirable to have a generic and flexible motor based vibrotactile testing system. To this end the authors designed a stimulator control program and a motor driving "controller-box" (Figure 7b) with which it interfaced.
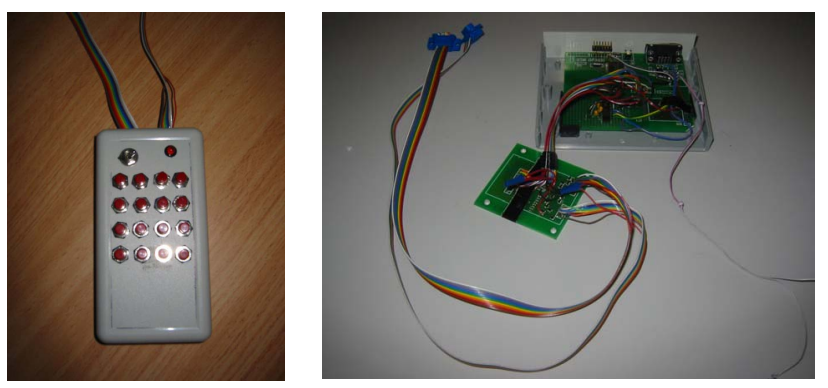

Figure 7: (a) "Button-Box" (b) "Controller-Box"

The "controller-box" is built around a TI MSP-430 microcontroller. A separate board is used to implement an array of Darlington amplifiers with kickback diodes to enable the microcontroller to drive the motors. The microcontroller interacts with the stimulator control program, a Microsoft Windows application used by the tester. The control program allows the tester to specify up to seven different states for motor firings. In each firing state the tester selects which motors are fired, how long all the selected motors fire, and the duration to wait between states. The resolution provided for the motor firing and wait states is in units of $25 \mathrm{~ms}$ and provides the tester a range of $0-254$ units. This provides the user the ability to create both fine grained and coarse testing patterns ranging in duration from $50 \mathrm{~ms}$ to $89 \mathrm{~s}$. Once a pattern is constructed and "fired" it is sent to the microcontroller for execution.

\section{Testing Apparatus}

Subjects tested the shoulder pads inserted into lined jackets in five sizes. The jacket size and shoulder pad size was chosen for each subject based on their neck-to-shoulder measurement. Subjects wore a standard 1-ply jersey knit cotton t-shirt underneath, with a thickness of $0.56 \mathrm{~mm}$. Jackets were donned first with conventional shoulder pads (those designed to go into the jackets) to allow the subjects to judge whether there were perceptible differences between standard and electronic shoulder pads.

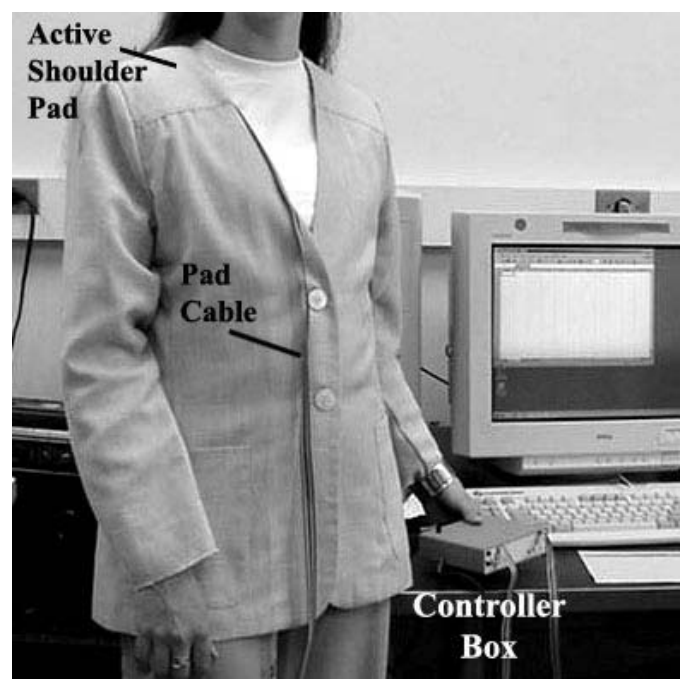

Figure 8: Testing Setup

Shoulder pads were constructed in two configurations, with either 4 or 6 motors. Motors were arranged in a t-shape, as shown in Figure 9.

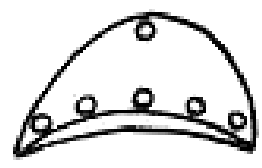

(a)

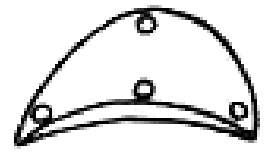

(b)
Figure 9: (a) Motor Locations for 6-motor configuration (b and 4-motor configuration

The pads were inserted into the jackets using hook-andloop $\left(\right.$ Velcro $\left.^{\circledR}\right)$ fasteners. Connecting wires protruded from the neckline edge of the shoulder pad, exiting the garment and falling down the front of the jacket to connect to the driving interface. Right and left shoulder pads have a mirrored motor numbering configuration. In both the 4 and the 6 motor configurations motor number 1 is the "frontmost" motor. Motor numbering then increases as the motors progress back over the shoulder. Figure 10 shows the motor numbering for both a 4 and 6 motor shoulder pads. 


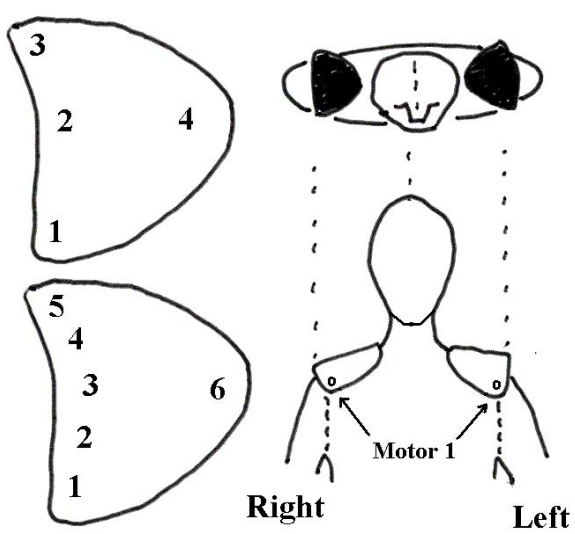

Figure 10: Motor Locations for left shoulder, for 4 and 6 motor configurations pictured against the Shoulder.

\section{Experimental Procedure}

\subsection{Subjects}

The 12 subjects were all female (to eliminate sex difference variables), between the ages of 19 and 34, with a variety of body types. Their shoulder lengths ranged from $9 \mathrm{~cm}$ to $14.5 \mathrm{~cm}$.

\subsection{Experimental Design}

Subjects were separated randomly into informed and uninformed groups to determine if prior knowledge of motor locations would influence perception abilities. These groups were then further subdivided randomly into a group testing the 6-motor configuration shoulder pad and a group testing the 4-motor configuration shoulder pad.

Both groups were first asked to don a test jacket in the appropriate size with standard shoulder pads. They then rated the comfort of the jacket in their relaxed standing position on a 5-point descriptive scale from very uncomfortable to very comfortable. The jacket was then removed and the shoulder pads replaced with two electronic shoulder pads. The subject rated the new configuration on the same comfort scale.

Uninformed subjects were not shown the motor configuration, but were only told that the electronic shoulder pads contained several vibrating motors, and that they would be asked to draw the area of their shoulder where they felt vibration.

Informed subjects were then shown the configuration of the motors within the electronic shoulder pad by showing them a shoulder pad with numbers showing the placement of each motor. They were told that motors would be activated in various combinations, and that they would be asked to draw the area on their shoulder where they felt vibration.

All subjects were tested by stimulating only the shoulder of their dominant hand. The vibrational stimulus was first presented by activation of all motors at once to orient them to the feeling of the motors generally. Once oriented, the testing consisted of a series of trials in which motors were activated first individually, then in pairs, in threes, and in fours, and finally fives and sixes for the 6-motor configuration groups.
Patterns were randomly ordered from a pre-determined set of patterns for each number of motors.

Each stimulus was activated for a period of 2.5 seconds. Subsequent patterns were activated after the subject finished drawing their response to the stimulus. Subjects recorded their responses to each trial on an illustration of a genderneutral body outline shown in Figure 11. They were told to indicate what they felt in a manner that would best communicate it, by drawing points, shaded areas, arrows or $\mathrm{x}$-marks, or any other depiction they felt was more suited to their experience.
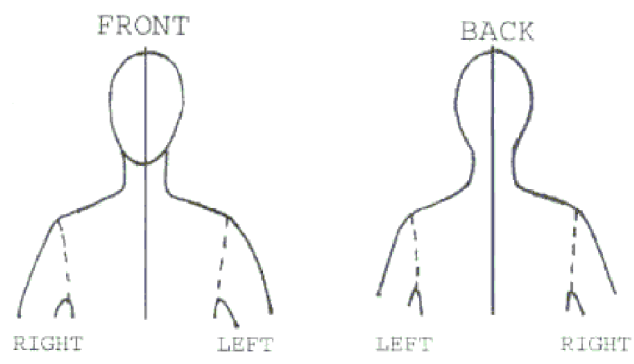

Figure 11: Subject Scoring Target

Following pattern trials, the subject was asked a series of qualitative questions to determine their general reaction to the tactile stimulus. They described the quality of the vibrational stimulus, the comfort level of the stimulus, and the amount of mental effort required to localize the origin of stimulus. The uninformed group was then debriefed and shown the actual location of tactors within the pads.

\section{Results}

\subsection{Analyzing the data}

The testing procedure required subjects to map perceived vibration onto a human torso, requiring the subjective mapping of perceived sensation onto the body outline shown in Figure 10.

In order to minimize any interpretational bias imposed by the authors in analysis of these data a blind and independent scorer mapped the user responses to motor positions. The scorer was provided a key of points across the shoulder that corresponded with motor location within the 4 and 6 motor configurations. For each subject the scorer was only informed as to which configuration (4 or 6 motors) was being tested and whether there were one or multiple motors active during each trial. Based on the subject responses, the scorer then recorded which motors appeared to be active. These results were then compared to the actual active motors for each trial. These data were then compiled and used for the analysis presented in this paper.

\subsection{Quantitative Results}

Perception: Most subjects' responses followed consistent patterns through their individual testing period although the perceived location of active motors varied from subject to subject. Subject response in mapping sensation onto the torso outline exhibited variation. Even provided with prior 
knowledge concerning the size and position of the shoulder pad the subjects reported a much wider range of perceived stimulation area then would be expected. Shown in Figure 12 the compilation of all user responses ranges unevenly down and to the side of the torso into the armpit.

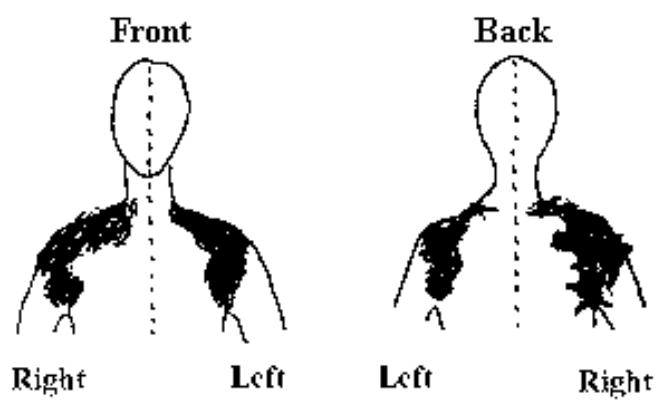

Figure 12: Composite User Responses

Detection: During testing, for $15 \%$ of the trials (where at least one motor was active) users reported the inability to detect any vibration. For the four motor configuration, this translates to detection of signal in $44 \%$ of 26 single motor firings, $13 \%$ of 47 double motor firings, and $0 \%$ of the three and four motor firings. For the six motor configuration, this translates to $27 \%$ of the 26 single motor firings, $15 \%$ of the 13 dual motor firings, $8 \%$ of the 25 triple motor firings and $0 \%$ of the four, five, and six motor firings.

Results identifying motors that subjects were consistently unable to detect were compiled and are shown in Figure 13. This compilation indicates the number of misses for tests firing one and two motors. Tests firing three or more motors have $100 \%$ detection for the forty randomized 4 motor configuration tests and $95 \%$ detection rate for 72 randomized tests of the 6 motor configuration.

All subjects experienced at least one motor that they consistently had difficulty feeling but the problematic motor varied across the subjects. All subjects could detect motor 2 in the four motor configuration (located at the outer shoulder tip) and motors 2 and 4 in the six motor configuration (located on the upper front and back of the outer shoulder edge).

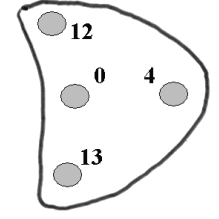

(a)

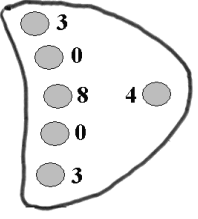

(b)
Figure 13: Miss Frequency for Each Motor Location

In many cases, the motors that could not be perceived individually were also not felt at all in the multiple-motor trials - these trials were perceived as if the missed motor(s) were not active. However, in some cases the missed motor contributed to the multiple-motor patterns, by extending the perceived area of stimulus in the direction of the missed motor.
The responses of the informed and uninformed groups were similar in both number and identity of motors that were not perceived. However, the informed group drew more focused areas of stimulus in their response diagrams.

\subsection{Qualitative responses}

As part of the study subjects were asked several open response questions. The external scorer read the open response comments and sorted them into categories reflecting the perceived quality of vibrational sensation, degree of comfort, concentration required, and ability to distinguish between sensation locations.

There was a large variation in the subject observations reported concerning the quality of the sensation they experienced. Subjects responded with comments ranging from comforting or soothing to annoying or ticklish, and one subject reported that some trials were painful. Grouping the subject comments on perceived degree of comfort with the vibration the external scorer rated 5 of the responses as showing a medium degree of comfort, seven as high, and one as very-low. The subjects' reports of the quality of the vibration are similarly distributed with 5 medium responses and 8 high responses.

A trend was observed in cognitive load that merits further testing; subjects who tested the 4-motor configuration in both groups (informed and uninformed) appeared to indicate a lower cognitive load than subjects testing the 6-motor configuration, based on their verbal responses to this question and on observation of their response time during the trials.

The user's perception of their ability to distinguish between sensation locations was generally low or very low. This subject perception appears to be in agreement with these data. Viewing just the single and dual firing tests we see only $32 \%$ and $34 \%$ perfect matches for the 4 and 6 motor configurations respectively.

The subjects generally reported no difference in comfort between the garment with normal shoulder pads and the garment with electronic shoulder pads. Three subjects reported an increased comfort level with the presence of the electronic shoulder pads.

\section{Discussion}

Given the small combined size of our subject population we have compiled data based on observations of our subjects and their response to the display. We do not have a large enough subject pool for rigorous statistical analysis, but we have successfully identified trends in the data for further study.

\subsection{Observations}

Generally the most-missed motors were those on the lowest and medial edges of the pads, those at the front and back axilla and at the intersection of the neck and shoulder, positions 1, 5, and 6 of six motor configuration, see Figure 13. This could be due to several factors: 1) the fit of the jacket in the shoulder area, which will influence the amount 
of pressure applied to the pad affecting the amount of skin contact; 2) the posture of the subject within the jacket, which can cause more or less pressure in an area of the shoulder; 3 ) the weight of the cables attached to the pad, which may pull the pad away from the body at the base of the neck; and 4) the fit of the shoulder pad, affecting the location of the axilla motors on the body.

The shoulder itself has a complex curved shape with much variation in the population. The ball of the humerus creates a convex curve in the front and back of the shoulder, the outermost edge of the shoulder curve. Underneath the ball joint, there is a concave hollow in the front of the body and a shallower convex curve in the back of the body. When the lower motors on the pad fall beneath the ball of the humerus, they are more likely to not be perceived, particularly in the front where the body curves away from the contour of the shoulder pad. The jacket construction does not provide a lateral force in to push the motor against the body.

Motor number 3 in the 6-motor configuration, which was not consistently perceived by subjects, corresponds in location to motor number 2 in the 4-motor configuration, which was always perceived by subjects. The six motor shoulder pad configuration is stiffer than the 4 motor shoulder pad configuration because of the proximity of the motors. The authors speculate that this increased stiffness may be responsible for the difficulty many subjects experienced in perceiving motor 3 in the six motor configuration, in contrast to motor 2 in the four motor configuration.

Misperceived motors on the pad edges often seemed to have an effect on the perception of multiple-motor patterns. One possibility is that the vibration of the motor is transmitted to some extent through the batting layers within the pad, and thus the mechanical coupling of the batting is felt by the subject at an intermediate point between motors where the body is in closer contact with the pad. The result is an expanded area of perceived stimulus.

\subsection{Sources of error}

Defining the shoulder: As Figure 12 shows, there was a very wide range of area that the subjects felt to constitute the shoulder. The authors propose two possible reasons as follows: 1) subject variation in mapping physical perception onto the body outline and/or 2) the vibrotactile display in conjunction with the garment produced a perceived tactile sensation away from the shoulder pad insert. Future work is required to understand variation in subject responses.

Scoring: The authors found that once the subjects had been familiarized with the operation of the shoulder pads and recorded some initial perceptions, the recorded responses generally increased in precision without any apparent corresponding increase in accuracy. The increase in subject precision was reflected in an increased number of marking locations and specificity of location. A corresponding increase in accuracy would have resulted in fewer missed or mischaracterized responses during testing. No such trend was found. The smaller sized markings meant a greater number of locations were chosen making the data hard to evaluate. As with the possible problem of mapping physical perception onto the body outline, improvements to the data collection regime are required for further studies.

\section{Proposed Design Guidelines}

The subjective perception of haptic interfaces is affected by several design considerations. Design issues are multiplied when the interface is embedded into a garment. The perceptibility of the interface is affected by the form and fit of the both the garment and the display device. In order to help facilitate further development in the area of shouldermounted tactile displays and garment-insert integrated electronics, the following guidelines are proposed:

- Vibro-tactile stimulators require garments to be constructed such that an even and adequate force is applied to the stimulators. The force bearing the stimulators' mechanical energy into the skin is related to garment weight, display device (shoulder pad) shape, and garment fit (taking into account subject posture).

- It is critical that garment embedded vibro-tactile stimulators remain free to vibrate along the axis normal to the curvature of the body. Garment construction that either places excessive hang weight upon the stimulator or encases the stimulator within a solid area will dampen or mute their vibrational capabilities.

- Display applications which require user perception of distinct activation areas increase the importance of ensuring that stimulators continually have the best possible contact with the body, even if additional garment layers come between the stimulator and the skin. Such applications therefore must either take into account optimal fit, or be equipped to apply a degree of pressure to the shoulder area, in order to ensure perception of active motors.

- Care must be taken that mechanical coupling of motors within display enclosure materials does not blur the boundaries of specific vibration regions.

- Shoulder-mounted garment integrated displays and their applications should strongly limit the number of discrete stimulating regions the user is expected to distinguish. Users seem able to accurately distinguish at least four discrete stimulation regions on one shoulder. However the number of discernable regions is directly affected by the proximity of the motors to the skin and the magnitude of motor vibration. The relative importance of these contributing factors seems to increase proportionally to the number of discernable stimulation regions.

\section{Future Work}

Key design issues remaining to be tackled include moving the stimulus closer to the body, optimizing number and placement of motors, optimizing garment weight, and examining the saltation phenomenon on the shoulder. The 
implementation of moving tactile stimuli on the shoulder to elicit physical movement for navigation or motion control will be investigated. Future wireless shoulder pads will allow for a more open response to testing and a broader range of subject responses and allow the authors to move from a static to dynamic testing paradigm.

Future applications of this kind of garment-integrated shoulder-mounted tactile display include assisted navigation, motion guidance, subtle communication alerts, and communication of low-bandwidth information. Possibilities also exist for investigation of applications that make use of the soothing nature of the vibrational sensation, in the areas of stress relief and biofeedback.

\section{Conclusions}

This work is the first to recognize that conventional clothing inserts offer an exciting new form factor for socially covert body worn devices. Device integration into standard garment inserts, such as the shoulder pad integrated tactile display demonstrated herein, allows intelligent clothing to be practically indistinguishable from conventional clothing while the device is not in use. Further design consideration can minimize the external perceptibility of the device during device usage. Ultimately these optimizations lead to clothingintegrated technology usable in the same manner as "off the rack" clothing, including with respect to care and cleaning.

Integration of devices into commercially viable clothing, particularly display devices, results in devices which cause minimal degradation to the social interaction that occurs between its user and others when not in use. According to the criteria outlined in [19] such garment-integrated devices would have and inherently low social weight.

This work is also the first to look at the shoulder with respect to haptic interfaces, covering several key issues surrounding shoulder mounted tactile interfaces. We have established initial guidelines for design and integration of a shoulder-mounted tactile display, initially documented a range in the level of user required concentration or cognitive load during device use, and further identified some factors in reducing this load for all users. Several trends in motor placement impacting perception of motors were also identified. Finally we have shown that such a shoulder worn tactile display can successfully make use of multiple stimulators, at a low level of resolution.

\section{References}

[1] Akamatsu, M. and MacKenzie, I. S. Movement characteristics using a mouse with tactile and force feedback. International Journal of Human Computer Studies, Vol. 45, pp 483-493, June 1996.

[2] Bach-y-RIta, P., Kaczmarek, K., Tyler, M., and Garcia-Lara, J. Form perception with a 49-point electrotactile stimulus array on the toung: A technical note. Journal of Rehabilitation Research and Development, Vol. 35, No. 4, pp 427-430, Oct 1998.

[3] Dunne, L. E., Ashdown, S. P., and McDonald, E. 'Smart Systems': Wearable Integration of Intelligent Technology. In International Center for Excellence in Wearable Computing and Smart Fashion Products, Cottbus, Germany, Dec 9-11, 2002.

[4] Edmison, J., Jones, M., Nakad, Z., and Martin, T. Using Piezoelectric
Materials for Wearable Electronic Textiles. In International Symposium on Wearable Computers, Seattle, WA, October 7-10,2002.

[5] Gemperle, F., Ota, N. K., and Siewiorek, D. Design of a Wearable Tactile Display. In International Symposium on Wearable Computing, Zurich, Switzerland, October, 2001.

[6] Gemperle, F., Kasabach, C., Stivoric, J., Bauer, M., and Martin, R. Design for Wearability. In International Symposium on Wearable Computers, pp 116-122, Pittsburgh,

[7] Gordon, C., Churchill, T., Clauser, C. E., Bradtmiller, B., T., M. J., Tebbetts, I., and Walker, R. A. Anthropometric Survey of U.S. Army Personnel: Methods and Summary Statistics. Technical Report, U.S. Army Natick Research \& Design Center, Natick, MA, Report No. Natick/TR-89/044, 1989,

[8] Gunther, E., Davenport, G., and Sile, O. M. Cutaneous Grooves: Composing for the Sense of Touch. In Conference on New Instruments for Musical Expression, Dublin, Ireland, May 24-26.

[9] Hayward, V. and Cruz-Hernandez, J. M. Tactile Display Device Using Distributed Lateral Skin Stretch. In Symposium on Haptic Interfaces for Virtual Environment and Teleoperator Systems,

[10]Lee, K. and Kwon, D.-S. Sensors and Actuators of Wearable Haptic Master Device for the Disabled. In International Conference on Intelligent Robots and Systems, pp 371-376, Takamatsu, Japan, Nov, 2000.

[11]Lindeman, R. W. and Cutler, J. R. Controller Design for a Wearable, Near-Field Haptic Display. In Haptic Interfaces for Virtual Environments and Teleoperator Systems, pp 397-403, 2003.

[12] May, K. W. and Selker, E. J. Integrated pointing device having tactile feedback. US, IBM, August 7, 2001.

[13] Montagu, A. Touching: The Human Significance of the Skin. New York, NY, Columbia University Press, 1971.

[14] Morikawa, O. Shoulder tapping illusion by vibrator in HyperMirror. In ICCS/JCSS99, pp 382-387, 1999.

[15]Nakamura, M. and Jones, L. An Actuator for the Tactile Vest, a TorsoBased Haptic Device. In Eleventh International Symposium on Haptic Interfaces for Virtual Environment and Teleoperator Systems, Los Angeles, CA, March 22-23, 2003.

[16] Rupert, A. H. An Instrumentation Solution for Reducing Spatial Disorientation Mishaps. IEEE Engineering in Medicine and Biology, pp 71-80, March/April.

[17] Tan, H. Z. and Pentland, A. Tactual displays for wearable computing. In pp 56-57, Cambridge, MA, October 13-14, 1997.

[18]Tan, H. Z., Ertan, S., Lee, C., Willets, A., and Pentland, A. A Wearable Haptic Navigation Guidance System. In International Symposium on Wearable Computing, Pittsburgh, PA, 1998.

[19] Toney, A., Mulley, B., Thomas, B. H., and Piekarski, W. Minimum Social Weight User Interactions for Wearable Computers in Business Suits. In International Symposium on Wearable Computers, Seattle, WA, October 7-10.

[20] Traylor, R. and Tan, H. Z. Development of a Wearable Haptic Display for Situation Awareness in Altered-Gravity Environment: Some Initial Findings. In International Symposium on Haptic INterfaces for Virtual Environments and Teleoperator Systems, Orlando, FL, 2002.

[21] Verrillo, R. T. Effect of contactor area on the vibrotactile threshold. J. Acoust. Soc. Am., Vol. 35, pp 1962-66, 1963.

[22] Verrillo, R. T. Vibrotactile thresholds for hairy skin. Journal of Exp. Psych, No. 72, pp 117-120, 1972.

[23] Verrillo, R. T., Fraioli, A. J., and Smith, R. L. Sensation magnitude of vibrotactile stimuli. Perception \& Psychophysics, Vol. 6, pp 366-372, 1969.

[24] Verrillo, R. T. and Chamberlain, S. C. The effect of neural density and contactor surround on vibrotactile sensation magnitude. Perception \& Psychophysics, Vol. 11, pp 117-120, 1972.

[25] Wall, C. I., Weinberg, M. S., Schmidt, P. B., and Krebs, D. E. Balance Prosthesis Based on Micromechanical Sensors Using Vibrotactile Feedback of Tilt. IEEE Transactions of Biomedical Engineering, Vol. 48, No. 10, pp 1153-61, October, 2001.

[26] Wilentz, J. S. The Senses of Man. NY, Thomas Y Crowell Company, 1968. 